\title{
Differential reinforcement effects on matching-order sequences
}

\author{
RICHARD PISACRETA \\ Ferris State College, Big Rapids, Michigan
}

\begin{abstract}
Three pigeons were previously trained on the matching-to-sample task with yellow, white, and blue stimuli. After acquisition, each of the three center-column keys of a nine-key response panel presented a different sample. The pigeons could match the three samples in any order. Then, reinforcement was provided only for particular sample matches, and later for matches on only one level (upper, middle, or lower keys). Intermittent reinforcement reduced matching accuracy. The birds frequently matched the only reinforced sample first. Later, they first matched whichever sample appeared on the only reinforced level. Additional novel-form stimuli reduced matching accuracy, and novel colors yielded some evidence of transfer of matching.
\end{abstract}

Recently, Pisacreta and Rautio (1984) trained pigeons on the matching-to-sample (MTS) paradigm with yellow, white, and blue stimuli. Each MTS trial was presented on the lower, middle, or upper three horizontal keys of a nine-key response panel. Later, each of the three center-column keys simultaneously presented a sample. The pigeons could match the samples in any order. The birds developed dominant matching-order sequences. The authors also reported that if different ratio values were paired with each sample, the birds would alter their matching-order sequences in order to match the samples with the lowest ratio requirement first. The birds also matched a "novel" sample last, when it was presented with two other "familiar" samples.

An interesting but seldom explored MTS manipulation is the use of intermittent reinforcement for correct matches. Nevin, Cumming, and Berryman (1963) reinforced correct matches on a fixed-ratio 3 (FR3), FR6, or FR9 schedule; that is, every third, sixth, or ninth correct match, respectively, produced grain. They reported an inverse relationship between the reinforcement ratio and matching accuracy. Furthermore, matching improved as the FR requirement was satisfied.

The first seven phases of the present experiment employed the concurrent MTS procedure used by Pisacreta and Rautio (1984) in conjunction with intermittent reinforcement for successful matches. This procedure was used for two purposes: (1) to see if intermittent reinforcement would reduce matching accuracy, and (2) to demonstrate that reinforcing only particular hue matches or correct matches on only one level (upper, middle, or lower keys) would control matchingorder sequences.

This study was supported by a faculty research grant to the author. Reprints may be obtained from Richard Pisacreta, Department of Psychology, Ferris State College, Big Rapids, MI 49307.

\section{METHOD}

\section{Subjects}

Three white Carneaux pigeons, maintained at $80 \%( \pm 15 \mathrm{~g})$ of their free-feeding weights, were used. The pigeons previously had been trained to match blue, white, and yellow stimuli when these colors were simultaneously presented on a nine-key response panel (see Pisacreta \& Rautio, 1984).

\section{Apparatus}

The apparatus was a $35 \times 35 \mathrm{~cm}$ operant chamber enclosed in a sound-attenuating hull. The response panel, $37 \times 35 \mathrm{~cm}$, had nine 2.7-cm (BRS/LVE Model 121-16) response keys arranged in a $3 \times 3$ matrix. Stimuli were rear-projected onto the keys by means of IEE in-line projectors. (See Pisacreta \& Rautio, 1984, for a more thorough description.) The feeder provided 3-sec access to grain. During sessions, illumination was provided only by the in-line projectors. A ventilation fan and white noise delivered through the speaker masked extraneous noises. An $\mathrm{E}$ and $\mathrm{L}$ Instruments MMD-1 computer and additional hardware recorded data and controlled experimental events.

\section{Procedure}

In Phase 1 (20 sessions), Keys 2, 5, and 8 each presented a different sample stimulus (white, yellow, and blue) at trial onset. A response to any sample extinguished the other two samples. Four more pecks (FR5) to the remaining sample extinguished it and lighted the two adjacent side keys with the comparison stimuli. A peck to the matching comparison key eliminated both comparison stimuli and produced the two remaining samples. After the second successful match, only the third sample was presented. A response to the matching comparison key produced a reinforcer, a 5 -sec intertrial interval, and the reillumination of three sample keys. Hence, only every third match was directly reinforced. An error (a peck to a nonmatching comparison stimulus) eliminated the comparison stimuli and reproduced all nonmatched samples. This correction procedure repeated a trial indefinitely until a correct match occurred. Although each bird had to successfully match each of the three samples, it could choose the order in which to match them. Sessions terminated after 36 correct matches had been produced.

During Phase 2 (10 sessions), only correct matches to the yellow samples were reinforced, whereas in Phase 3 (20 sessions), only correct matches to the blue samples were reinforced. In Phase 4 (15 sessions), only matches to white were reinforced. In Phase 5 (15 sessions), only correct matches emitted on Keys 1 , 2 , and 3 , the upper level, were reinforced. In Phase 6 (15 ses- 
sions), only correct matches on Keys 7, 8, and 9, the lower level, were reinforced. Then, during Phase 7 (20 sessions), only correct matches emitted on Keys 4, 5, and 6, the middle level, produced grain. In Phase 8 (15 sessions), continuous reinforcement (CRF) was provided for all correct matches.

During Phase 9 ( 3 sessions), the sample stimuli were yellow, white, and a novel white square presented on a black background. In Phase 10 ( 3 sessions), the stimuli available for matching were yellow, square, and a novel horizontal white line presented on a black background. In Phase 11 ( 3 sessions), three forms (square, horizontal line, and vertical line) were available, whereas during Phase 12 (5 sessions), three colors (white, red, and green) were available for matching. Finally, the baseline combination of blue, yellow, and white stimuli were employed for Phase 13 (3 sessions).

\section{RESULTS}

Figure 1 shows the matching levels generated during Phases 1-8, inclusive. The first panel of Figure 1 shows that each bird maintained $80 \%-95 \%$ matching accuracies even though only every third match was reinforced. Birds 1, 2, and 3 (B1, B2, and B3) averaged five, six, and four errors per session, respectively. Reinforcement of particular stimulus matches, Phases 2-4, reduced matching accuracies in all birds. The majority of errors were the result of each bird's pecking the incorrect comparison stimulus that was also the only stimulus being reinforced when it served as the sample. During Phase 2 , in which only yellow matches produced food, B1, B2, and B3 made $67 \%, 80 \%$, and $69 \%$ of their errors, respectively, by pecking incorrect yellow comparison stimuli (i.e., the sample was blue or white). Likewise, during Phase 3 , in which only blue matches were reinforced, B1, B2, and B3 emitted $68 \%, 76 \%$, and $70 \%$ of their errors, respectively, by erroneously pecking blue comparison stimuli during white and yellow MTS trials.

After 15 sessions of Phase 3, B3 failed to complete sessions. The bird matched blue and yellow samples but stopped responding when white was the only remaining sample. Each daily session terminated after $30 \mathrm{~min}$

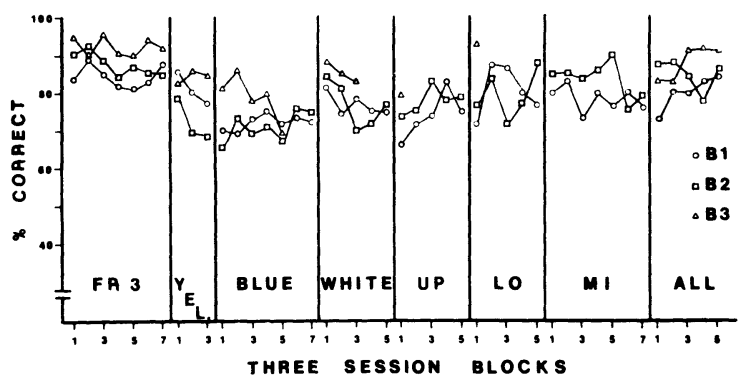

Figure 1. Percentage of correct matches generated during each three-session block of Phases 1-8, inclusive. Reinforced matches are denoted at the bottom of each panel. The panels (from left to right) indicate that reinforcement was provided only for: every third match, yel(low) matches, blue matches, white matches, up(per) level matches, lo(wer) level matches, mi(ddle) level matches, and, finally, all sample matches and levels. without a response. Similarly, B3 stopped completing sessions after the ninth, fourth, third, and second sessions of Phases 4, 5,6, and 7, respectively.

During Phase 4 , in which only white matches were reinforced, $74 \%, 68 \%$, and $75 \%$ of B1's, B2's, and B3's errors, respectively, were to white comparison stimuli. During Phases 5, 6, and 7, in which there was differential reinforcement of upper, lower, and middle level matches, respectively, the errors were evenly distributed among the blue, yellow, and white comparison stimuli, and on all three levels. During Phases 2-7, inclusive, B1, B2, and B3 averaged 25, 22, and 16 errors per session, respectively. For B1 and B2, matching improved during Phases 5, 6, and 7, in which each sample was reinforced four times each session. Each bird yielded matching accuracies during Phase 8 that were comparable to those levels produced during other sessions that provided continuous reinforcement for each correct match.

Figure 2 depicts the percentage of the trials that each bird responded to the only reinforced sample or key level as its first match. Panel 1, Phase 2, shows that when only yellow matches produced food, each bird matched yellow first (instead of blue or white) during $80 \%$ $90 \%$ of the trials. Panel 2, Phase 3 , in which only blue matches were reinforced, shows that the birds' firstmatch preferences shifted from yellow matches to blue matches within 10 sessions. The remaining four panels of Figure 2 show that the birds shifted their matching preferences to coincide with the available reinforcers. Each bird matched the sample, or the level, that yielded a reinforcer, and then completed the remaining two unreinforced matches.

Figure 3 shows the matching performances produced during Phases 8-13, inclusive, in which various novel stimuli were provided. Panel 1 , Phase 8 , shows that each bird produced matching levels comparable to those yielded when blue, white, and yellow stimuli were employed before. During Phase 9 (square, white, and yellow stimuli) and Phase 10 (square, horizontal line, and yellow stimuli), each bird maintained an abovechance matching level. During Phase 9, $85 \%, 88 \%$, and $90 \%$ of B1's, B2's, and B3's errors, respectively, were on trials in which the square or white stimulus was an incorrect comparison stimulus. Similarly, during Phase 10, $100 \%, 92 \%$, and $99 \%$ of B1's, B2's, and B3's errors, respectively, were on trials in which the sample and comparison stimuli were both forms. During Phase 11, B1's and B3's matching performances dropped below chance levels during the sessions with three form stimuli. During these sessions, B1, B2, and B3 averaged 40, 19, and 29 errors per session, respectively. Introducing two novel color stimuli (red and green) produced an immediate recovery in matching accuracy. The matching levels of Phase 13 replicated those of Phase 8.

Table 1 shows the dominant key-order and stimulusorder matching sequences emitted during Phases 8-13, 


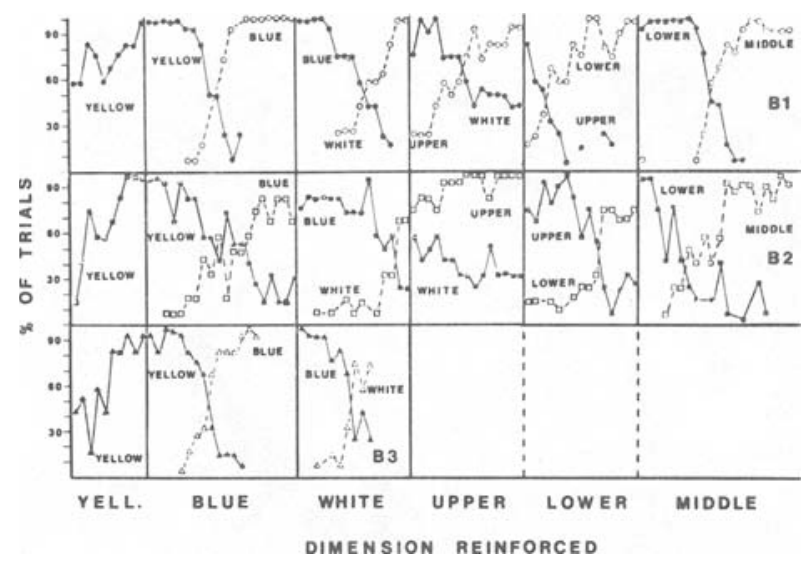

Figure 2. Percentage of trials per session on which each bird responded to the only reinforced sample (Phases 2, 3, and 4) or level (Phases 5, 6, and 7) first. Filled data points represent the reinforced dimension from each previous phase. Unfilled data points display the reinforced dimension within the phase.

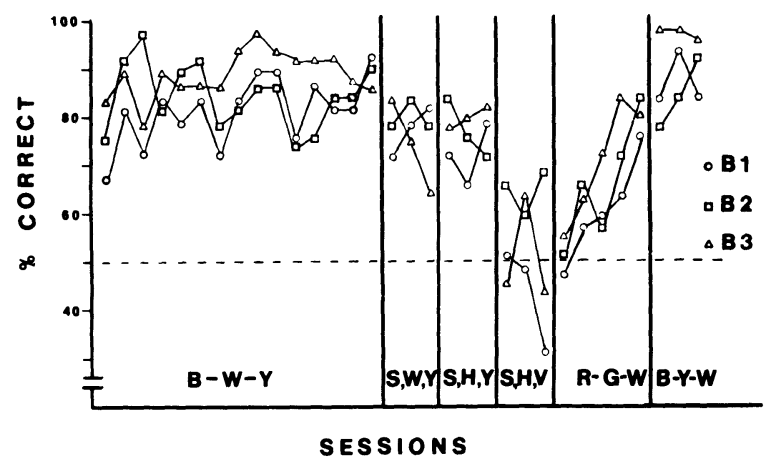

Figure 3. Percentage of correct matches produced during each session of the last six phases (Phases 8-13). The dotted line represents chance performance. The stimuli included: B(lue), W(hite), Y(ellow), S(quare), H(orizontal line), V(ertical line), $R(e d)$, and $G($ reen).

Table 1

Dominant Key-Order and Stimulus-Order Sequences

\begin{tabular}{|c|c|c|c|c|c|c|c|}
\hline \multirow[b]{2}{*}{ Phase } & \multirow[b]{2}{*}{ Samples } & \multicolumn{2}{|c|}{ B1 } & \multicolumn{2}{|l|}{ B2 } & \multicolumn{2}{|l|}{ B3 } \\
\hline & & Order & $\%$ & Order & $\%$ & Order & $\%$ \\
\hline \multirow[t]{2}{*}{8} & B-Y-W & $8-5-2$ & 73 & $5-2-8$ & 37 & $8-5-2$ & 48 \\
\hline & & & & $5-8-2$ & 37 & $8-2-5$ & 40 \\
\hline \multirow[t]{2}{*}{9} & S-Y-W & $8-5-2$ & 75 & $5-2-8$ & 36 & $8-2-5$ & 67 \\
\hline & & & & $5-8-2$ & 36 & $8-5-2$ & 30 \\
\hline \multirow[t]{2}{*}{10} & S-Y-H & $8-5-2$ & 64 & $8-5-2$ & 33 & $8-5-2$ & 47 \\
\hline & & & & $5-8-2$ & 33 & $8-2-5$ & 39 \\
\hline \multirow[t]{2}{*}{11} & S-V-H & $8-5-2$ & 64 & $8-5-2$ & 78 & $8-2-5$ & 50 \\
\hline & & & & & & $8-5-2$ & 22 \\
\hline \multirow[t]{2}{*}{12} & R-G-W & $8-5-2$ & 67 & W-G-R & 48 & W-R-G & 53 \\
\hline & & & & W-R-G & 37 & R-W-G & 37 \\
\hline \multirow[t]{2}{*}{13} & B-Y-W & $8-5-2$ & 92 & W-Y-B & 39 & $8-5-2$ & 72 \\
\hline & & & & $5-8-2$ & 36 & Y-W-B & 31 \\
\hline
\end{tabular}

Note-Dominant sequences generated during Phases 8-13, inclusive. The samples included: B(lue), Y(ellow), W(hite), $S$ (quare), H(orizontal line), R(ed), and G(reen). inclusive. Table 1 indicates that each bird predominantly used dominant sample key-order sequencès during Phases $8,9,10$, and 11. During Phase 12, in which novel colors were introduced for matching and matching accuracy recovered, B2 and B3 employed dominant sample-order matching sequences, and B1's dominant key-order sequence was maintained. The dominant sequences emitted in Phase 13 were similar to those produced during Phase 8, which offered the same matching conditions.

\section{DISCUSSION}

The concurrently available sample procedure appears to be a useful technique for studying complex learning and the relative strength of several conditional discriminations. Trilevel samples enable pigeons to show stereotyped response sequences similar to those reported by Pisacreta (1982a, 1982b, 1982c). Multiple available samples also provide an opportunity for pigeons to initially avoid novel sample stimuli, and show that differential reinforcement contingencies can easily manipulate matching sequence orders. There is little doubt that pigeons acquire conditional discriminations. The $80 \%-90 \%$ matching accuracies support this conclusion. The present data, however, may indicate that stimulus control by these conditional discriminations, present in the MTS paradigm, is weaker than is supposed. During Phases 2, 3, and 4, only yellow, blue, and white matches, respectively, were reinforced with grain. Most errors during those phases were pecks to the nonmatching comparison stimulus that also served as the only sample that could produce grain, that is, errors to yellow, blue, and white comparison stimuli, in Phases 2, 3 , and 4 , respectively. These decreases in matching performance occurred even though each bird had previously successfully completed 17,000 matching trials. Specifically, selective reinforcement disrupted established matching behavior. Similar results were reported by Paul (1983).

The present work appears to support the multiple-rule model, or matching as a case of several two-component chains position (see Carter \& Werner, 1978). In the present case, the behaviors "if blue is the sample; then peck the blue comparison stimulus," "if yellow is the sample, then yellow," and "if white, then white," were maintained provided each match produced reinforcement. Reinforcing only one match seemed to occasion the chain "peck the sample and then peck the only comparison stimulus that is associated with food." Even the correction procedure, repeating a trial indefinitely until a correct match occurs, did not suppress this tendency. If conditional discriminations in general, and MTS in particular, are a matter of response chaining in nonhumans, it appears to be weak chaining at best.

Finally, presenting three or more simultaneous samples may provide additional insight into the mechanisms involved when nonhumans acquire complex associative discriminations.

\section{REFERENCES}

Carter, D. E., \& Werner, T. J. (1978). Complex learning and information processing by pigeons: A critical analysis. Journal of the Experimental Analysis of Behavior, 29, 565-601.

Nevin, J. A., Cumming, W. W., \& Berryman, R. (1963). Ratio reinforcement of matching behavior. Journal of the Experimental Analysis of Behavior, 6, 149-154.

Paul, C. (1983). Ratio effects in matching-to-sample. Journal of the Experimental Analysis of Behavior, 39, 77-85.

Pisacreta, R. (1982a). Concurrent sequential matching in the pigeon. Bulletin of the Psychonomic Society, 20, 183-186.

Pisacreta, R. (1982b). Preferences among stimulus matches in 
the pigeon. Journal of the Experimental Analysis of Behavior, 38, 191-199.

Pisacreta, R. (1982c). Some factors that influence the acquisition of complex, stereotyped responses in pigeons. Journal of the Experimental Analysis of Behavior, 37, 359-369.
Pisacreta, R., \& Rautio, J. (1984). Effects of sample-specific ratios on free-choice matching-order sequences. Bulletin of the Psychonomic Society, 22, 244-247.

(Revision received for publication July 2, 1984.) 